\title{
PASTA-PHASE TRANSITIONS IN THE INNER CRUST OF NEUTRON STARS*
}

\author{
X. Viñas ${ }^{\mathrm{a}}$, C. Gonzalez-Boquera ${ }^{\mathrm{a}}$, B.K. Sharma ${ }^{\mathrm{b}}$, M. Centelles $^{\mathrm{a}}$ \\ ${ }^{a}$ Departament de Física Quàntica i Astrofísica \\ and \\ Institut de Ciències del Cosmos (ICCUB), Facultat de Física \\ Universitat de Barcelona, Martí i Franqués 1, 08028 Barcelona, Spain \\ ${ }^{\mathrm{b}}$ Department of Sciences, Amrita School of Engineering \\ Amrita Vishwa Vidyapeetham, Amrita University, 641112 Coimbatore, India
}

\section{(Received January 10, 2017)}

We perform calculations of nuclear pasta phases in the inner crust of neutron stars with the Thomas-Fermi method and the Compressible Liquid Drop Model using the Barcelona-Catania-Paris-Madrid (BCPM) energy density functional and several Skyrme forces. We compare the crust-core transition density estimated from the crust side with the predictions obtained from the core by using the thermodynamical and dynamical methods. Finally, the correlation between the crust-core transition density and the slope of the symmetry energy at saturation is briefly analyzed.

DOI:10.5506/APhysPolBSupp.10.259

\section{Introduction}

Neutron stars (NS) are among the most fascinating objects of the universe. They are ultradense and compact remnants formed at the last stage of life of massive stars [1,2]. An NS is composed by neutrons, protons, leptons and eventually other exotic particles distributed in a solid crust encompassing a dense core in a liquid phase. The full NS is electrically neutral and the distribution of nucleons and leptons is such that the system is maintained locally in $\beta$-equilibrium. The structure of the crust consists of clusters of positive charge distributed in a solid lattice embedded in a bath of neutrons and free electrons. This complicated structure is often referred to as a "nuclear pasta" and makes the derivation of the Equation of State (EOS) in this region a difficult task, in particular, in the inner crust owing to the

* Presented at the XXIII Nuclear Physics Workshop "Marie and Pierre Curie", Kazimierz Dolny, Poland, September 27-October 2, 2016. 
presence of a fraction of free neutrons. The formation and properties of nuclear pasta have been investigated through 3-dimensional Hartree-Fock (HF), relativistic mean field (RMF) and Thomas-Fermi (TF) calculations in cubic boxes, which avoid assumptions about their geometry. Unfortunately, these calculations are so time consuming that it is difficult to obtain with them a detailed EOS for the whole inner crust (see [3] for recent references to such calculations). Large scale calculations of the inner crust and nuclear pasta have often been performed with the help of semiclassical methods such as the Compressible Liquid Drop Model (CLDM) [4-6] and the TF approach $[3,7,8]$. Our aim in this contribution is twofold. First, we want to discuss the pasta phases present in the inner crust of NS obtained through TF and CLDM calculations. To this end, we employ the recent BCPM energy density functional [9-11], which consists of a fully microscopic bulk part obtained from the Brueckner-Hartree-Fock calculations including three-body forces plus a phenomenological finite range term of Gaussian type, and some Skyrme forces. Second, we want also to analyze the transition density between the crust and the core of NS. Although large scale calculations of the inner crust could provide such densities, there are not many calculations of this type available in the literature. Therefore, it is easier to go in the opposite direction and examine the stability conditions of the core against small-amplitude density fluctuations using the so-called thermodynamical and dynamical methods (see [12,13] for references and more details). The paper is organized as follows. In the first part, we briefly revise the TF and CLDM approaches for describing pasta phases. In a second part, we estimate the transition density from the crust side using several Skyrme forces and compare with the predictions obtained from the core side. Finally, we give our conclusions in the last section.

\section{Thomas-Fermi approximation to the neutron star inner crust}

In this approximation, as well as in the CLDM, the crust is treated at the Wigner-Seitz (WS) level assuming a single nuclear cluster inside a cell with a given geometry, which is electrically neutral and does not interact with other WS cells. The total energy of an ensemble of neutrons, protons and electrons in a WS cell of volume $V_{\mathrm{c}}$ is given by $[3,7,14]$

$$
\begin{aligned}
E= & \int_{V_{\mathrm{c}}} \mathrm{d} V\left[\mathcal{H}\left(n_{n}, n_{p}\right)+\mathcal{E}_{\text {elec }}+\mathcal{E}_{\text {Coul }}-\frac{3}{4}\left(\frac{3}{\pi}\right)^{1 / 3} e^{2}\left(n_{p}{ }^{4 / 3}+n_{e}{ }^{4 / 3}\right)\right. \\
& \left.+m_{n} n_{n}+m_{p} n_{p}\right],
\end{aligned}
$$

where $n_{n}, n_{p}$ and $n_{e}$ are the neutron, proton and electron number densities, and $m_{n}$ and $m_{p}$ are the neutron and proton masses. The nuclear energy 
density $\mathcal{H}\left(n_{n}, n_{p}\right)$ in the TF approach reads

$$
\mathcal{H}\left(n_{n}, n_{p}\right)=\frac{3}{5}\left(3 \pi^{2}\right)^{2 / 3}\left[\frac{\hbar^{2}}{2 m_{n}} n_{n}^{5 / 3}(\boldsymbol{r})+\frac{\hbar^{2}}{2 m_{p}} n_{p}^{5 / 3}(\boldsymbol{r})\right]+\mathcal{V}\left(n_{n}(\boldsymbol{r}), n_{p}(\boldsymbol{r})\right)
$$

In this equation, $\mathcal{V}\left(n_{n}(\boldsymbol{r}), n_{p}(\boldsymbol{r})\right)$ is the nuclear potential energy density, which in this work we take from the BCPM and Skyrme models. At the densities prevaling in the inner crust of NS, the Fermi energy is much higher than the Coulomb energy and, therefore, we can treat the electrons as a free relativistic Fermi gas of energy density $\mathcal{E}_{\text {elec }}$ uniformly distributed in the cell with a constant density $n_{e}$. The Coulomb energy density coming from the direct part of the proton-proton, electron-electron and proton-electron interactions is given by

$$
\begin{aligned}
\mathcal{E}_{\text {Coul }} & =\frac{1}{2}\left(n_{p}(\boldsymbol{r})-n_{e}\right)\left(V_{p}(\boldsymbol{r})-V_{e}(\boldsymbol{r})\right) \\
& =\frac{1}{2}\left(n_{p}(\boldsymbol{r})-n_{e}\right) \int \frac{e^{2}}{\left|\boldsymbol{r}-r^{\prime}\right|}\left(n_{p}\left(\boldsymbol{r}^{\prime}\right)-n_{e}\right) \mathrm{d} \boldsymbol{r}^{\prime}
\end{aligned}
$$

where $V_{p}(\boldsymbol{r})$ and $V_{e}(\boldsymbol{r})$ are the proton and electron direct Coulomb potentials.

We perform a fully variational calculation of the energy in a WS cell of size $R_{\mathrm{c}}$ under the constraints of a given average density $n_{b}$ and charge neutrality. Taking functional derivatives with respect to the neutron, proton and electron densities, one finds

$$
\begin{aligned}
\frac{\delta \mathcal{H}\left(n_{n}, n_{p}\right)}{\delta n_{n}}+m_{n}-\mu_{n} & =0, \\
\frac{\delta \mathcal{H}\left(n_{n}, n_{p}\right)}{\delta n_{p}}+V_{p}(\boldsymbol{r})-V_{e}(\boldsymbol{r})-\left(\frac{3}{\pi}\right)^{1 / 3} e^{2} n_{p}^{1 / 3}(\boldsymbol{r})+m_{p}-\mu_{p} & =0, \\
\sqrt{k_{\mathrm{F}}^{2}+m_{e}^{2}}-V_{p}(\boldsymbol{r})+V_{e}(\boldsymbol{r})-\left(\frac{3}{\pi}\right)^{1 / 3} e^{2} n_{e}^{1 / 3}-\mu_{e} & =0,
\end{aligned}
$$

together with the $\beta$-equilibrium condition imposed by the aforementioned constraints

$$
\mu_{e}=\mu_{n}-\mu_{p},
$$

where $\mu_{e}, \mu_{n}$ and $\mu_{p}$ are the electron, neutron and proton chemical potentials, respectively. 
For a given average density $n_{b}$ and a size $R_{\mathrm{c}}$ of the WS cell, we obtain the composition and energy per baryon in this cell by solving the above equations. Next, we perform a search of the optimal size $R_{\mathrm{c}}$ to find the absolute minimum of the energy per baryon for the average density $n_{b}$. The EOS is provided by the sum of pressures of the neutron and electron gases plus a corrective term due to the electron Coulomb exchange [3,7]. The same TF method used for spherical WS cells can be easily extended to deal with other geometries, such as the cylindrical (rods) and the planar (slabs) ones. The length of the rods and the area of the slabs are considered infinite, which simplifies the treatment of the Coulomb interaction and provides a finite energy per baryon. With a suitable choice of the initial conditions, the same TF formalism can describe hollow spheres (bubbles) and cylinders (tubes). In summary, the TF method is a reasonable tool to estimate the EOS in the inner crust of NS by two main reasons. First, the EOS in this region is largely driven by the neutron gas, therefore, it is expected that the contributions to the EOS due to shell effects and pairing correlations play a minor role. Second, as far as shell effects are neglected in the TF method, it is well-suited for dealing with non-spherical shapes of the WS cells.

The self-consistent TF approximation has been applied recently to obtain the EOS of the inner crust of NS [3,7] using the BCPM energy density functional [9-11]. The energy per baryon corresponding to different shapes, relative to the value of uniform npe matter, is displayed in figure 1, where the bottom of the inner crust is displayed in two panels in order to appreciate the appearance of the different pasta phases. The order in which the different phases appear is consistent with earlier literature [2]. In our calculation using the BCPM functional, the shape transition from droplets to rods appears at a density of $0.067 \mathrm{fm}^{-3}$, from rods to slabs at $0.076 \mathrm{fm}^{-3}$, from slabs to tubes at $0.082 \mathrm{fm}^{-3}$ and, finally, from tubes to bubbles almost at the transition density to the core, which is found at $0.0825 \mathrm{fm}^{-3}$.
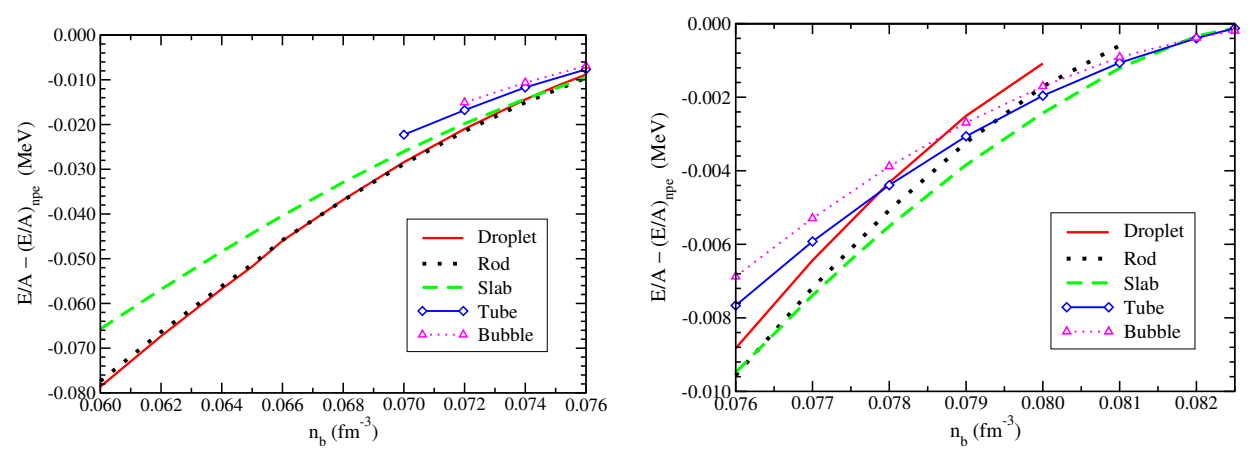

Fig. 1. Energy per baryon of different shapes relative to uniform npe matter as a function of the baryon density in the inner crust. Adapted from Ref. [3]. 


\section{Compressible Liquid Drop Model}

The CLDM was introduced by Baym, Bethe and Pethick [4], see also [6] and references therein. One assumes that each WS cell contains two phases of asymmetric nuclear matter in equilibrium. The phase of highest density, which fills a fraction $\chi$ of the total volume of the cell $V_{\mathrm{c}}$, is formed by neutrons and protons and corresponds to the nuclear cluster. The phase of lowest density, which fills the remaining volume of the cell, is associated to the neutron gas. Charge neutrality is provided by a uniform free electron gas filling the whole cell. The energy in the cell is the sum of the bulk energies of the cluster and the neutron and electron gases supplemented by the repulsive surface energy of the cluster and the Coulomb proton and electron self-interactions plus the attractive proton-electron contribution. The corresponding energy density, constrained to a given average baryon density $n_{b}$, is given by

$$
\begin{aligned}
\varepsilon= & \chi \varepsilon\left(n_{n}, n_{p}\right)+(1-\chi) \varepsilon\left(n_{\mathrm{d}}, 0\right)+\frac{3 \sigma \chi}{R} \\
& +\frac{4 \pi}{5} e^{2} R^{2} n_{p}^{2} \chi\left(1-\frac{3}{2} \chi^{1 / 3}+\frac{1}{2} \chi\right)+\frac{3}{4}\left(3 \pi^{2}\right)^{1 / 3} n_{e}^{4 / 3} \\
& -\lambda\left[\chi\left(n_{n}+n_{p}\right)+(1-\chi) n_{\mathrm{d}}-n_{b}\right],
\end{aligned}
$$

where $n_{n}$ and $n_{p}$ are the neutron and proton densities in the cluster and $n_{\mathrm{d}}$ is the neutron density in the dripped gas. The surface tension $\sigma$ in (8) is a function of the average density $n_{b}$ and, in our calculation, is taken from a self-consistent extended TF calculation performed in semi-infinite nuclear matter [15]. Taking variations with respect to the variables $n_{n}, n_{p}, R$ and $R_{\mathrm{c}}$, which means requiring mechanical and chemical equilibrium among the different subsystems, one arrives at the following set of equations:

$$
\begin{aligned}
\lambda & =\mu_{n}=\frac{\partial \varepsilon\left(n_{n}, n_{p}\right)}{\partial n_{n}}=\frac{\partial \varepsilon\left(n_{\mathrm{d}}, 0\right)}{\partial n_{\mathrm{d}}}, \\
\mu_{n}-\mu_{p}-\mu_{e} & =\frac{8 \pi}{5} e^{2} R^{2} n_{p}\left(1-\frac{3}{2} \chi^{1 / 3}+\frac{1}{2} \chi\right), \\
P_{\mathrm{i}}-P_{\mathrm{o}} & =\frac{2 \sigma}{R}-\frac{4 \pi}{15} e^{2} R^{2} n_{p}^{2}(1-\chi), \quad\left(E_{\text {surf }}=2 E_{\text {Coul }}\right), \\
4 \pi R^{2} \sigma & =\frac{32 \pi^{2}}{5} e^{2} R^{5} n_{p}\left(1-\frac{3}{2} \chi^{1 / 3}+\frac{1}{2} \chi\right), \\
n_{b} & =\chi\left(n_{n}+n_{p}\right)+(1-\chi) n_{\mathrm{d}},
\end{aligned}
$$

which allow one to determine the unknowns $n_{n}, n_{p}, n_{\mathrm{d}}, R$ and $\chi$. The first equation implies the equality of the neutron chemical potential in the bulk 
and in the neutron gas. The second equation corresponds to the $\beta$ equilibrium, modified with respect to the uniform case by the effect of the lattice. The third and fourth equations concern the mechanical equilibrium. The third one gives the difference of pressures between the cluster and the gas due to the surface and the lattice and the fourth one, known as "virial theorem", determines the equilibrium size of the WS cell, which involves only the Coulomb and surface contributions. The total pressure in a WS cell is given by $P=P_{\mathrm{o}}+P_{\mathrm{L}}+P_{e}$, where $P_{\mathrm{o}}$ is the pressure due to the neutron gas, $P_{\mathrm{L}}=-\partial E_{\text {Coul }} / \partial V_{\mathrm{c}}$ is the lattice pressure with $E_{\text {Coul }}$ being the Coulomb energy, and $P_{e}$ is the contribution of the electrons. The CLDM approach can be easily extended to cylindrical and plane geometries. The nuclear bulk and electron contributions are shapeindependent but the surface and Coulomb terms depend on the dimensionality $d$ as $\varepsilon_{\text {surf }}=\frac{\chi d}{R}\left[\left(n_{n}-n_{\mathrm{d}}\right) \mu_{n} s_{n}+\sigma\right]$ and $\varepsilon_{\text {Coul }}=\frac{4 \pi}{5}\left(n_{p} e R\right)^{2} f_{d}(\chi)$, respectively, where $f_{d}(\chi)=\frac{5}{d+2}\left[\frac{1}{d-2}\left(1-\frac{1}{2} \chi^{1-2 / d}\right)+\frac{1}{2} \chi\right]$ if $d=1$ or 3 and $f_{d=2}=\frac{5}{8}(\chi-1-\ln \chi)$. In the case of holes (tubes and bubbles), one has to change the sign of the surface term and replace $\chi$ by $1-\chi$ (see Ref. [2] for more details).

In figure 2 , we display the total number of nucleons $\left(A_{\text {cell }}\right)$ and protons $\left(Z_{\text {cluster }}\right)$ present in spherical WS cells against the average density $n_{b}$. The number of neutrons in the cluster $\left(N_{\text {cluster }}\right)$ and in the gas $\left(N_{\text {gas }}\right)$ are also shown. The results are obtained with the SLy4 Skyrme interaction using the self-consistent TF approximation and an improved CLDM, which includes neutron skin thickness corrections and curvature and surface width corrections [16]. It can be seen that there is an excellent agreement between the predictions of both semiclassical methods. The total number of nucleons in the cluster, $A_{\text {cluster }}$, increases monotonically from $\sim 120$, when neutrons start to drip, till $\sim 200$, when the transition to the core takes place. However, the proton number in the clusters remains rather constant, around 42. The number of neutrons in the gas increases up to $\sim 1000$ at an average density close to $0.02 \mathrm{fm}^{-3}$ and then decreases up to $\sim 800$ at the edge of the inner crust. A similar trend is followed by the total number of nucleons in the cell, which are largely driven by the neutron gas. These behaviors of the clusters and the neutron gas are quite general and the predictions of other Skyrme forces will follow a similar tendency. We have solved the CLDM equations corresponding to the different shapes using the SLy4, MSL0, SkX and UNEDF1 Skyrme forces. For these forces, the spherical droplet is the most stable shape up to densities around $0.075 \mathrm{fm}^{-3}$, where non-spherical geometries become the preferred shapes, which indicates the appearance of pasta phases. For the mentioned Skyrme forces, nuclear pasta only appears in the case of the SkX force, whose transition density of $0.086 \mathrm{fm}^{-3}$ is high enough to allow non-spherical shapes as the most stable configurations. 

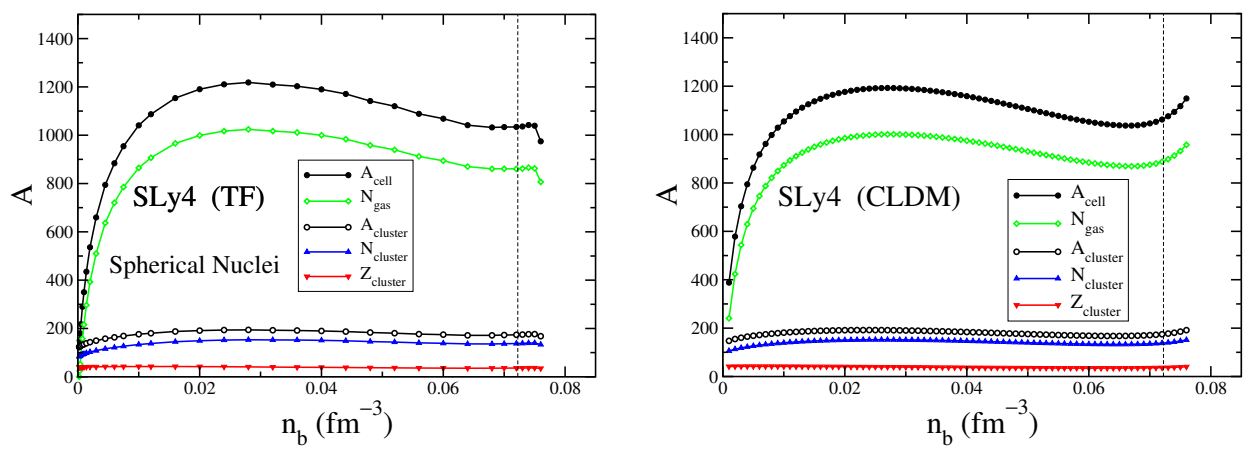

Fig. 2. Mass and atomic numbers in spherical WS cells as a function of the average density predicted by the SLy4 interaction using the TF approach and the CLDM.

\section{The crust-core phase transition}

The bottom of the inner crust is reached when a homogeneous phase, consisting of neutrons, protons and electrons filling uniformly the whole WS cell, becomes energetically more stable than the possible phases of the inner crust. The determination of the transition density from the crust side requires large scale calculations. Even using simplified methods such as the TF or CLDM described in the previous sections, these calculations are rather time consuming and in practice there are not many calculations of this type available in the literature $[3,5,6,8]$. To avoid this problem, the crust-core phase transition has been also studied from the core side. There are mainly three well-established methods, namely the thermodynamical method [17], the dynamical method $[18,19]$ and the random phase approximation (for more details, see $[12,13]$ and references therein). These methods are based on the onset of violation of the stability conditions of the homogeneous core against small-amplitude density fluctuations, which indicates the formation of inhomogeneous nuclear structures, i.e. the appearance of the inner crust. The thermodynamical method corresponds to the long wavelength limit of the dynamical method and both give similar results. The transition density predicted by the dynamical method is slightly smaller due to the inclusion of Coulomb effects and inhomogeneities in the density [12].

In figure 3, we display the crust-core transition density, computed with the thermodynamical and dynamical methods, as a function of slope of the symmetry energy $L$ for a large set of Skyrme interactions. The transition density shows, roughly, a linear decreasing trend with increasing value of $L$, in agreement with the findings in earlier literature [12]. In the same figure, we show the transition density predicted by the CLDM calculations in the inner crust using the Skyrme interactions considered in the previous section. The CLDM transition densities are $0.065,0.071,0.072$ and $0.086 \mathrm{fm}^{-3}$ with the MSL0, UNEDF1, SLy4 and SkX forces, respectively. These values can be 
compared with the dynamical estimates, which for the same interactions are $0.070,0.078,0.080$ and $0.094 \mathrm{fm}^{-3}$, respectively. We see that, roughly, there is a difference of about $10 \%$ between the estimates from the crust and core sides (also see [19]). These differences may be related to the approximations used to compute the transition density. For example, estimating the crustcore transition density with the more elaborated TF approach, one obtains a value of $0.076 \mathrm{fm}^{-3}$ using the SLy4 force, which is closer to the dynamical estimate than the CLDM prediction. When the transition density is high enough, it is possible that the pasta shapes appear before the spherical nuclei dissolve [20]. Thus, the decreasing tendency of the transition density with the slope of the symmetry energy and the discussion of the previous section also suggest that the appearance of pasta phases in the inner crust may be favored by a soft symmetry energy, which implies a relatively high transition density.

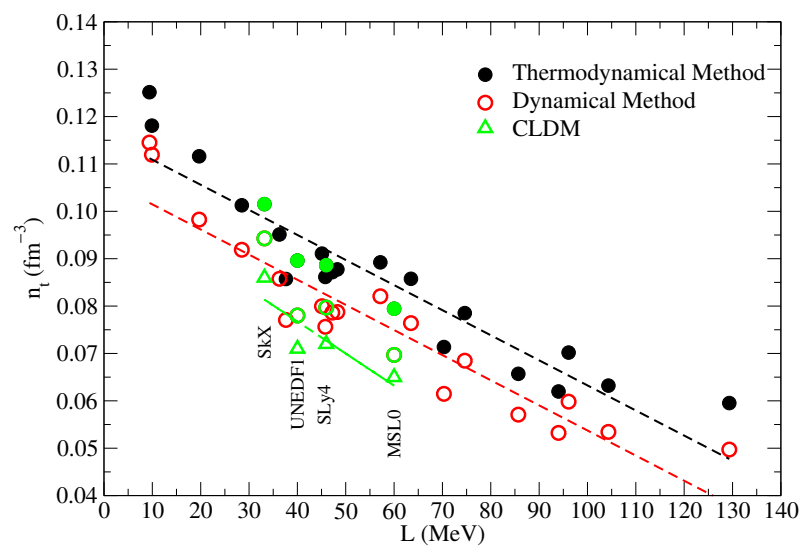

Fig. 3. Transition density against the slope of the symmetry energy for several Skyrme forces, calculated using the thermodynamical (filled dots) and the dynamical (unfilled dots) methods in the core, and using the CLDM (unfilled triangles) in the crust. Gray/green symbols correspond to the Skyrme forces used in Sec. 3.

\section{Conclusions}

We have analyzed the shape transitions in the inner crust of neutron stars using the self-consistent Thomas-Fermi approximation and the Compressible Liquid Drop Model. These semiclassical methods allow to study in a rather simple way not only conventional spherical droplets but also other geometries such as cylinders, plates, tubes and bubbles, which simulate complicated pasta phases in the inner crust. The study of these pasta phases is very delicate from a numerical point of view due to the extremely 
small differences among the energies per baryon computed in the different geometries. It is found that calculations performed with the Compressible Liquid Drop Model are in a good agreement with the predictions of the Thomas-Fermi approximation.

We have also studied the crust-core phase transition from the core side through the thermodynamical and dynamical methods, which estimate the density of the core that becomes unstable against small-amplitude fluctuations. Proceeding from the crust side and using the Compressible Liquid Drop Model, we have determined the transition density as the value for which the homogeneous phase is the most favorable one from an energetic point of view. It is found that the estimates of the transition density from the crust and core sides are in harmony. The transition density determined in both ways follows a decreasing tendency with the slope of the symmetry energy. This correlation also suggests that the appearance of pasta phases may be favored by nuclear models with soft symmetry energy.

Work partially supported by grants FIS2014-54672-P from MINECO and FEDER, 2014SGR-401 from Generalitat de Catalunya and MDM-2014-0369 ICCUB (Unidad de Excelencia María de Maeztu) from MINECO. C.G. also acknowledges grant BES-2015-074210 from MINECO.

\section{REFERENCES}

[1] S.L. Shapiro, S.A. Teukolsky, Black Holes, White Dwarfs and Neutron Stars: The Physics of Compact Objets, Wiley-Interscience, New York 1983.

[2] P. Haensel. A.Y. Potekhin, D.G. Yakovlev, Neutron Stars 1: Equation of State end Structure, Springer, New York 2006.

[3] B.K. Sharma et al., Astron. Astrophys. 584, A103 (2015).

[4] G. Baym, H.A. Bethe, C.J. Pethick, Nucl. Phys. A 175, 225 (1971).

[5] J.M. Lattimer, D.F. Swesty, Nucl. Phys. A 535, 331 (1991).

[6] F. Douchin, P. Haensel, Astron. Astrophys. 380, 151 (2001).

[7] M. Baldo et al., Phys. At. Nucl. 77, 1157 (2014).

[8] H. Shen, H. Toki, K. Oyamatsu, K. Sumiyoshi, Nucl. Phys. A 637, 435 (1998); Prog. Theor. Phys. 100, 1013 (1998).

[9] M. Baldo, P. Schuck, X. Viñas, Phys. Lett. B 663, 390 (2008).

[10] M. Baldo, L.M. Robledo, P. Schuck, X. Viñas, J. Phys. G 37, 064015 (2010).

[11] M. Baldo, L.M. Robledo, P. Schuck, X. Viñas, Phys. Rev. C 84, 064305 (2013).

[12] J. Xu, L.W. Chen, B.A. Li, H.R. Ma, Astrophys. J. 697, 1549 (2009).

[13] T.R. Routray et al., J. Phys. G 43, 105101 (2016). 
[14] T. Sil et al., Phys. Rev. C 66, 045803 (2002).

[15] M. Centelles, M. Del Estal, X. Viñas, Nucl. Phys. A 635, 193 (1998).

[16] B.K. Sharma, C. Gonzalez-Boquera, M. Centelles, X. Viñas, work in progress.

[17] S. Kubis, Phys. Rev. C 70, 065804 (2004); 76, 025801 (2006).

[18] G. Baym, C.J. Pethick, P. Sutherland, Astrophys. J. 170, 299 (1971).

[19] F. Douchin, P. Haensel, Phys. Lett. B 485, 107 (2000).

[20] Ch.C. Moustakidis, Phys. Rev. C 86, 015801 (2012). 